EPJ Web of Conferences 43, 03003 (2013)

DOI: $10.1051 /$ epjconf/20134303003

(C) Owned by the authors, published by EDP Sciences, 2013

\title{
Red giant seismology: Observations
}

\author{
B. Mosser ${ }^{\mathrm{a}}$
}

LESIA, CNRS, Université Pierre et Marie Curie, Université Denis Diderot, Observatoire de Paris, 92195 Meudon Cedex, France

\begin{abstract}
The CoRoT and Kepler missions provide us with thousands of red-giant light curves that allow a very precise asteroseismic study of these objects. Before CoRoT and Kepler, the red-giant oscillation patterns remained obscure. Now, these spectra are much more clear and unveil many crucial interior structure properties. For thousands of red giants, we can derive from seismic data precise estimates of the stellar mass and radius, the evolutionary status of the giants (with a clear difference between clump and RGB stars), the internal differential rotation, the mass loss, the distance of the stars... Analyzing this amount of information is made easy by the identification of the largely homologous red-giant oscillation patterns. For the first time, both pressure and mixed mode oscillation patterns can be precisely depicted. The mixed-mode analysis allows us, for instance, to probe directly the stellar core. Fine details completing the red-giant oscillation pattern then provide further information on the interior structure, including differential rotation.
\end{abstract}

\section{INTRODUCTION}

The CNES CoRoT mission [1] and the NASA Kepler mission [2] have opened a new era in red giant asteroseismology [3], with thousands of high-precision photometric light curves. This amount of data has motivated collaborative working in dedicated working groups, with an organization very profitable for promoting efficient work and impressive results [e.g. 4-7]. This paper has benefited from all this work.

Before space-borne observation, ground-based observations have revealed that red giants, with an outer convective envelope, show solar-like oscillations [e.g. 8]. Owing to their low gravity, oscillations in red giants are excited at low frequency, and because of their low mean density, their oscillation pattern shows frequency differences, called the large separation, which is of the order of a few microhertz. Limitations due to too short observing runs (even if the longest lasted about two months) and to a poor duty cycle have hampered a rich output of these ground-based observations but raised crucial questions concerning the degree of the observed modes and the mode lifetimes. [8] explicitly state that "a most important and exciting result of [their] study is the confirmation of the possibility, suggested by the results reported on $\alpha \mathrm{UMa}$ and Arcturus, to observe solar-like oscillations in stars on the red giant branch". These questions were not answered by observations with the microsatellite MOST [e.g. 9], with time series limited to one month. However, the pioneering role of these observations was highly valuable, so that red giants were considered as important asteroseimic targets. Without them, both CoRoT and Kepler would have missed an impressive harvest.

In Section 2, we first present results obtained when considering global seismic parameters only. Such parameters allow us to perform ensemble asteroseismology. The tools for identifying the individual frequencies are then presented in Section 3. The identification of the dipole mixed-mode pattern, not as

\footnotetext{
ae-mail: benoit.mosser@obspm.fr
}

This is an Open Access article distributed under the terms of the Creative Commons Attribution License 2.0, which permits unrestricted use, distribution, and reproduction in any medium, provided the original work is properly cited. 


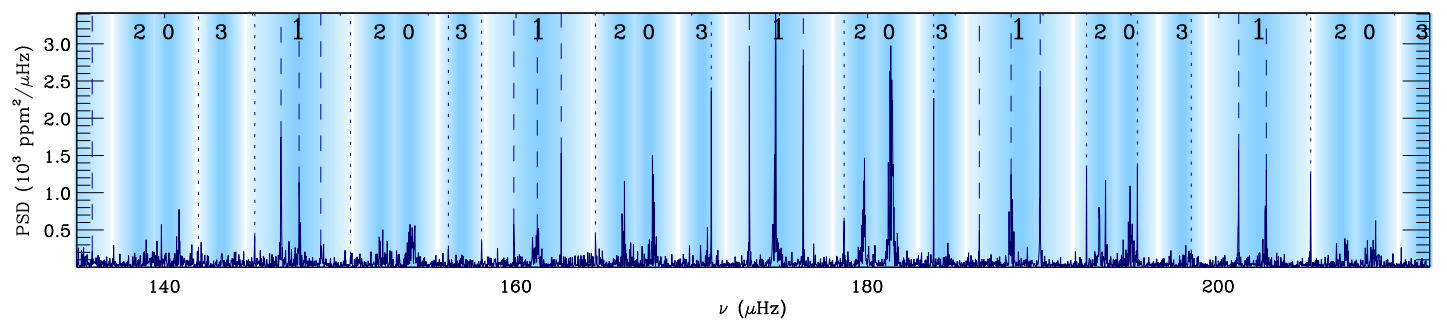

Figure 1. Power density spectrum of the star KIC 9882316, with superimposed mode identification provided by the red giant oscillation universal pattern. Dashed and dotted lines indicate the position of the peaks identified as dipole mixed modes. Pressure dominated dipole modes are located close to the positions marked by 1.

easily achievable as for the radial pressure mode pattern, is developed in Section 4. These mixed modes unveil unique properties of the core. Open questions and upcoming work are presented in Section 5.

An introduction to red giant seismology, by [10] and [11] for theoretical aspects or by [12] in an observational perspective, can be useful for setting the scene.

\section{SCALING RELATIONS}

A large amount of scaling relations have been recently derived in asteroseismology [e.g. 4, 13, 14]. Such relations are based on global seismic parameters used to sum up the mean properties of a solarlike oscillation spectrum. They allow us to perform ensemble asteroseismology, since they monitor the evolution of various parameters for a large population of stars.

\subsection{Global seismic parameters}

Most scaling relations involve the large separation $\Delta v$ and/or the frequency $v_{\max }$ corresponding to the maximum oscillation signal. The determination of these global seismic parameters can be done diversely [see e.g. 15]. Here, we use essentially the data analysis provided by the method of [16], called envelope autocorrelation function (EACF). Deriving the large separation from the autocorrelation of the time series is physically efficient, since it corresponds to measuring the delay between any oscillation signal first seen directly, then after propagation throughout the stellar diameter back and forth. Achieving this autocorrelation of the time series of the oscillation signal by computing the Fourier transform of its Fourier transform of the oscillation signal is computationally very efficient. Considering a windowing of the spectrum, as proposed by [17], allows us to select a given frequency range, or to investigate the variation of the frequency separations with frequency, or to study independently the frequency separations of the even and odd ridges [18]. With a filter width corresponding to the frequency range around $v_{\max }$ where solar-like oscillations are excited, the method provides the mean value of the observed large separation. Last but not least, the methodology used by the EACF method provides a test for determining the reliability of the detection, based on the H0 hypothesis.

Scaling relation in asteroseismology is an old story, when [19] noted that the pulsations of cepheids are related to their mean density. This can be expressed by the following scaling relation

$$
\Delta v \propto \sqrt{\frac{M}{R^{3}}}
$$

where $\Delta v$ is the mean large separation, and $M$ and $R$ are the stellar mass and radius. $\Delta v$ is usually defined as the mean frequency difference between consecutive radial modes (Fig. 1). In fact, this definition is misleading: frequency differences yield the observed value of the large separation, which is different 


\section{Ageing Low Mass Stars: From Red Giants to White Dwarfs}

from the asymptotic value that verifies Eq. (1). The link between the large separation and the mean stellar density has been addressed by [20] for different stellar masses and evolutionary stages. The relation between the observed and asymptotic values of the large separation is established by [21]:

$$
\Delta v_{\mathrm{as}}=(1+\zeta) \Delta v_{\mathrm{obs}},
$$

with

$$
\begin{gathered}
\left.\zeta=\frac{0.57}{n_{\max }} \quad \text { (main-sequence regime: } n_{\max } \geq 15\right), \\
\left.\zeta=0.038 \quad \text { (red giant regime: } n_{\max } \leq 15\right),
\end{gathered}
$$

where $n_{\max }=v_{\max } / \Delta v$ measures the frequency of maximum power of oscillation signal in a dimensionless manner. The relation between $v_{\max }$ and the acoustic cutoff frequency $v_{\mathrm{c}}$ proposed by [22] introduces the Mach number $\mathcal{M}$ in the uppermost convective layers so that $v_{\max } \propto v_{\mathrm{c}} \mathcal{M}^{3}$. The variation of this number with stellar type and evolution is limited but remains unknown.

\subsection{Seismic mass and radius}

The importance of the measurements of $\Delta v$ and $v_{\max }$ is emphasized by their ability to provide relevant estimates of the stellar mass and radius

$$
\begin{gathered}
\frac{R_{\text {seis }}}{R_{\odot}}=\left(\frac{v_{\text {max }}}{v_{\text {ref }}}\right)\left(\frac{\Delta v_{\mathrm{as}}}{\Delta v_{\text {ref }}}\right)^{-2}\left(\frac{T_{\text {eff }}}{T_{\odot}}\right)^{1 / 2}, \\
\frac{M_{\text {seis }}}{M_{\odot}}=\left(\frac{v_{\text {max }}}{v_{\text {ref }}}\right)^{3}\left(\frac{\Delta v_{\text {as }}}{\Delta v_{\text {ref }}}\right)^{-4}\left(\frac{T_{\text {eff }}}{T_{\odot}}\right)^{3 / 2} .
\end{gathered}
$$

The reference value $\Delta v_{\text {ref }} \simeq 3106 \mu \mathrm{Hz}$ and $v_{\text {ref }} \simeq 138.8 \mu \mathrm{Hz}$ have been determined by [21], relying on the exact use of the second-order asymptotic expression and on the calibration with modeled stars. Unbiased estimates of $R$ and $M$ are provided only if the asymptotic value of the large separation is used. The use of the observed large separation induces significant bias, of the order of $3 \%$ for the radius and $6 \%$ for the mass.

Even if the calibration effort is not complete, the scaling relations give relevant estimates. [21] have shown that the correct use of the scaling relations with the asymptotic large separation provides estimates of $R$ and $M$ with uncertainties of about 4 and $8 \%$, respectively, for low-mass stars. Uncertainties are twice larger when $M \geq 1.3 M_{\odot}$ or for red giants.

\subsection{Ensemble asteroseismology}

Scaling relations on global parameters allow us to perform ensemble asteroseismology.

- The radius-mass diagram puts in evidence the mass-loss occurring at the tip of the red giant branch (RGB, Fig. 2). The mass loss of low-mass stars is strong enough to reduce the mass of their envelope to less than $0.2 M_{\odot}$.

- [24] have performed a comparative study of the granulation background in giants. The parameters of the background in the Fourier spectrum are closely related to the parameters of the solar-like oscillation, so that a mechanism able to partition the convective energy between oscillation and granulation must exist. For instance [23] have shown that the height-to-background ratio at $v_{\max }$ is constant for red giants, with only a slight difference between RGB and clump stars.

- Scaling relations of the oscillation amplitude were reported by different groups [e.g. 7, for CoRoT observations], from main-sequence stars to red giants [14] or using red giants in clusters [25]. Previous models have failed to reproduce the scaling relations. With 3D hydrodynamical models representative 


\section{EPJ Web of Conferences}

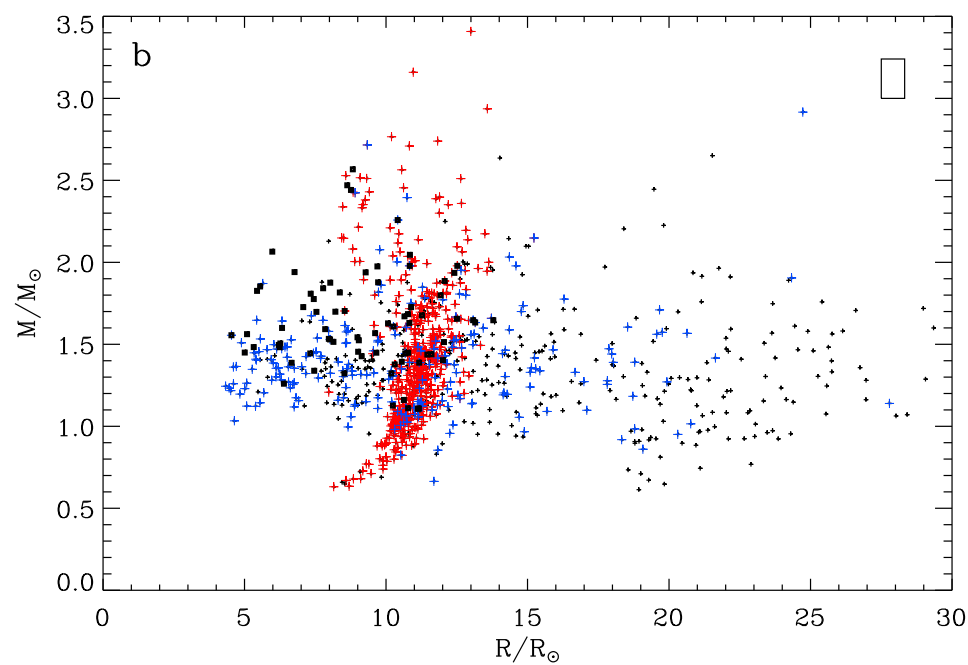

Figure 2. Asteroseismic mass as a function of asteroseismic radius. The color code indicates the evolutionary status; clump stars in red, giant branch stars in blue, unknown status in dark grey. The population of giants with low $\ell=1$ amplitude is indicated with black squares. The rectangles in the upper right corners indicate the mean value of the $1 \sigma$ error bars. From [23].

of the upper layers of sub- and red giant stars, the acoustic mode energy supply rate computed by [26] shows that scaling relations of mode amplitudes cannot be extended from main-sequence to red giants because non-adiabatic effects for red giant stars cannot be neglected.

\section{FREQUENCY PATTERN}

Any person involved in the data analysis of red giants rapidly gets the impression that all red giant spectra are very similar. This has to be related to the fact that red giants have necessarily very similar interiors. Before evolving into a red giant, the star has undergone the exhaustion of hydrogen in its core, the contraction of its helium core, the separation of the continuously contracting core from the continuously growing envelope, with a thin hydrogen-burning shell at the interface, and the growth of a large convective envelope. All these steps, mostly governed by the properties of the hydrogen-burning shell (equation of state, power supply rate), have erased most of the original characteristics of the stars. After the tip of the RGB, all low-mass red giants pass through the helium flash. As a consequence, they gain a new opportunity to reach almost the same interior structure, as shown by the mass-radius relation of clump stars (Fig. 2).

[27] have capitalized this necessary similarity to set up a method for measuring very precisely the large separation and for identifying red-giant oscillation spectra in an automated way. Assuming that these oscillations obey to a universal pattern, they have proposed that the offset $\varepsilon$ of the asymptotic relation [28] is a function of the large separation. They have expressed the second-order term of the asymptotic relation with a quadratic term that relates the curvature of the ridges observed in the échelle diagrams:

$$
v_{n, \ell}=\left[n+\frac{\ell}{2}+\varepsilon\left(\Delta v_{\mathrm{obs}}\right)-d_{0 \ell}\left(\Delta v_{\mathrm{obs}}\right)+\frac{\alpha_{\ell}}{2}\left(n-n_{\max }\right)^{2}\right] \Delta v_{\mathrm{obs}} .
$$

We use here the subscript $o b s$ to emphasize the difference with the asymptotic value. The different $d_{0 \ell}$ terms indicate the small spacings of non-radial modes [27]. [29] have shown that $\alpha_{0}$ is also a function of the large separation. This implies that all curvatures $\alpha_{\ell}$ depend on $\Delta v_{\text {obs }}$. This method has proven to be 
efficient for all red giants, with a large separation in the range [0.4 - 40 $\mu \mathrm{Hz}]$, especially for oscillation spectra recorded with a low signal-to-noise ratio.

The univocal relation between $\varepsilon_{\mathrm{obs}}$ and $\Delta v_{\mathrm{obs}}$, updated by [30], is insured if the large separation is observed in a large frequency range. When determined in a limited frequency range, the small difference of the offset $\varepsilon$ between RGB and clump stars allows us to determine the evolutionary status of the giant [31].

[21] have recently shown that the relation $\varepsilon\left(\Delta v_{\mathrm{obs}}\right)$ is an artefact, so that the radial modes of red giants follow the pattern:

$$
\begin{aligned}
v_{n, 0} & =\left(n+\frac{1}{4}+0.037 \frac{n_{\max }^{2}}{n}\right) \Delta v_{\mathrm{as}} \\
& =\left(n+\frac{1}{4}+\frac{18.3}{n}\left(\frac{M}{M_{\odot}} \frac{R_{\odot}}{R} \frac{T_{\odot}}{T_{\mathrm{eff}}}\right)\right) \Delta v_{\mathrm{as}} .
\end{aligned}
$$

based on the asymptotic value $\Delta v_{\text {as }}$ of the large separation. This equation is fully equivalent to Eq. 7 , when taken into account the relation between the asymptotic and observed values of the large separation provided by Eq. 2.

Departures to such a regular spectrum are due to rapid structure discontinuities. They induce socalled glitches in the oscillation spectrum, as due to the second ionization of helium [32]. [33] have shown that an asymptotic development can be used to address the signature of such a discontinuity. However, the red giant oscillation spectrum is also much more complex, due to the presence of other oscillation modes than pure pressure modes.

\section{MIXED MODES AND STELLAR EVOLUTION}

\subsection{Stellar evolution}

[34] have identified mixed modes in an RGB star. Such mixed modes result from pressure waves propagating in the envelope coupled with gravity waves trapped in the core. Due to the contraction of the inert helium core, the Brunt-Väisälä frequency reaches much higher values than in main-sequence stars, so that the coupling between the different waves in the envelope and in the core is efficient [e.g. 11]. This coupling permits the information of gravity modes to percolate to the surface. Hence, [35] could show that the mixed-mode frequency separation depends on the evolutionary status of the star and allows to distinguish helium-burning stars in the red clump from shell hydrogen-burning stars in the RGB. [36] have proposed an alternative method, based on the EACF with narrow filters centered on the dipole modes. These first approaches only deliver the bumped period spacing, significantly perturbed by the coupling of the pressure and gravity waves and quite different from the period spacing $\Delta \Pi_{1}$ of gravity modes.

\subsection{Asymptotic development of the mixed mode pattern}

The period spacing $\Delta \Pi_{1}$ is derived from the asymptotic development for mixed modes exposed by [37], based on the method of [38]. Observations of red giant with a large number of dipole mixed modes give rise to this development. The mixed-mode frequencies related to the pure pressure dipole mode of radial order $n$ are solutions of the implicit equation:

$$
v=v_{n, \ell=1}+\frac{\Delta v}{\pi} \arctan \left[q \tan \pi\left(\frac{1}{\Delta \Pi_{1} v}-\varepsilon_{\mathrm{g}}\right)\right]
$$

where $v_{n, \ell=1}$ is the pure pressure mode frequency previously determined, $q$ is a dimensionless coupling factor, $\Delta \Pi_{1}$ is the period spacing of pure gravity modes and $\varepsilon_{\mathrm{g}}$ is a constant fixed to 0 . For each pressure radial order $n$, one obtains $\mathcal{N}+1$ solutions, with $\mathcal{N} \simeq \Delta v \Delta \Pi_{1}^{-1} v_{\max }^{-2}$. The value of $\Delta \Pi_{1}$ is derived from 


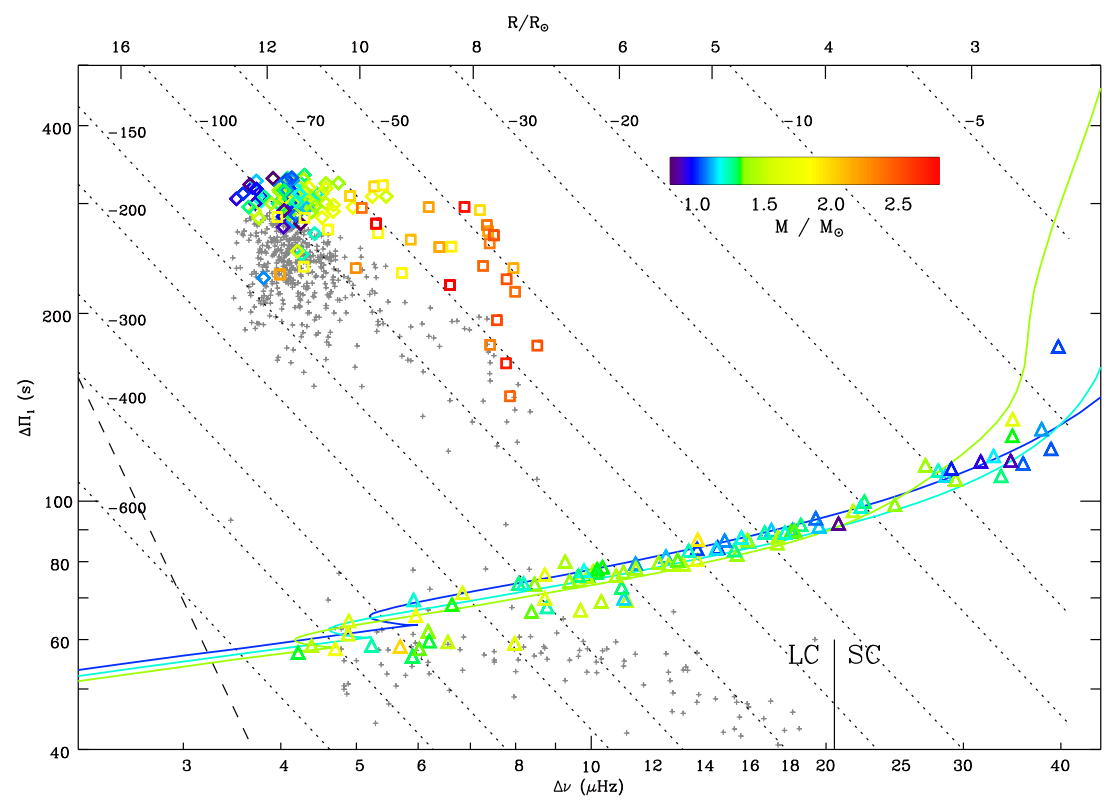

Figure 3. Gravity-mode period spacing $\Delta \Pi_{1}$ as a function of the pressure-mode large frequency spacing $\Delta \nu$. Longcadence data (LC) have $\Delta v \leq 20.4 \mu \mathrm{Hz}$. RGB stars are indicated by triangles; clump stars by diamonds; secondary clump stars by squares. Uncertainties in both parameters are smaller than the symbol size. The seismic estimate of the mass is given by the color code. Small gray crosses indicate the bumped periods $\Delta P_{\text {obs }}$ measured by [36]. Dotted lines are $n_{\mathrm{g}}$ isolines. The dashed line in the lower left corner indicates the formal frequency resolution limit. The upper $\mathrm{x}$-axis gives an estimate of the stellar radius for a star whose $v_{\max }$ is related to $\Delta v$ according to the mean scaling relation $v_{\max }=(\Delta v / 0.28)^{1.33}$ (both frequencies in $\mu \mathrm{Hz}$ ). The solid colored lines correspond to a grid of stellar models with masses of $1,1.2$ and $1.4 M_{\odot}$, from the ZAMS to the tip of the RGB. From [37].

a least-squares fit of the observed values to the asymptotic solution. As shown by [37], the observation of high gravity mode orders insures a precise description of the mixed-mode pattern with the asymptotic development. As a result, the period $\Delta \Pi_{1}$ can be determined with a high accuracy (Fig. 3). This is highly valuable to directly characterize the stellar cores [11].

For low-mass stars on the RGB, the close relationship between the large separation $\Delta v$ and the period spacing $\Delta \Pi_{1}$ emphasizes the homology of red giants (Fig. 3). This underlines the fact that the properties of the stellar envelope are completely governed by the properties of the helium core and its hydrogen-burning shell.

\subsection{Rotational splittings}

[39] have shown that gravity-dominated mixed modes reveal the core rotation in red giants. They analyzed the rotational splittings of three red giant oscillation spectra, in the early stages of the RGB. These splittings reveal a significant differential rotation, with a core rotating at least ten times faster than the surface.

[29] have developed a method for analyzing rotation splittings in an automated way, based on the EACF function with ultra-narrow filters. This method has provided splittings in more than 260 red giants observed with Kepler. A direct identification of the rotational splittings, provided by the method proposed by [37], was also performed for more than 100 red giants (Figs. 4, 5). Under the hypothesis that a linear analysis can provide the mean core rotation from the rotational splittings of the gravitydominated mixed modes, the evolution of this mean core rotation indicates that a significant spin down 
Ageing Low Mass Stars: From Red Giants to White Dwarfs
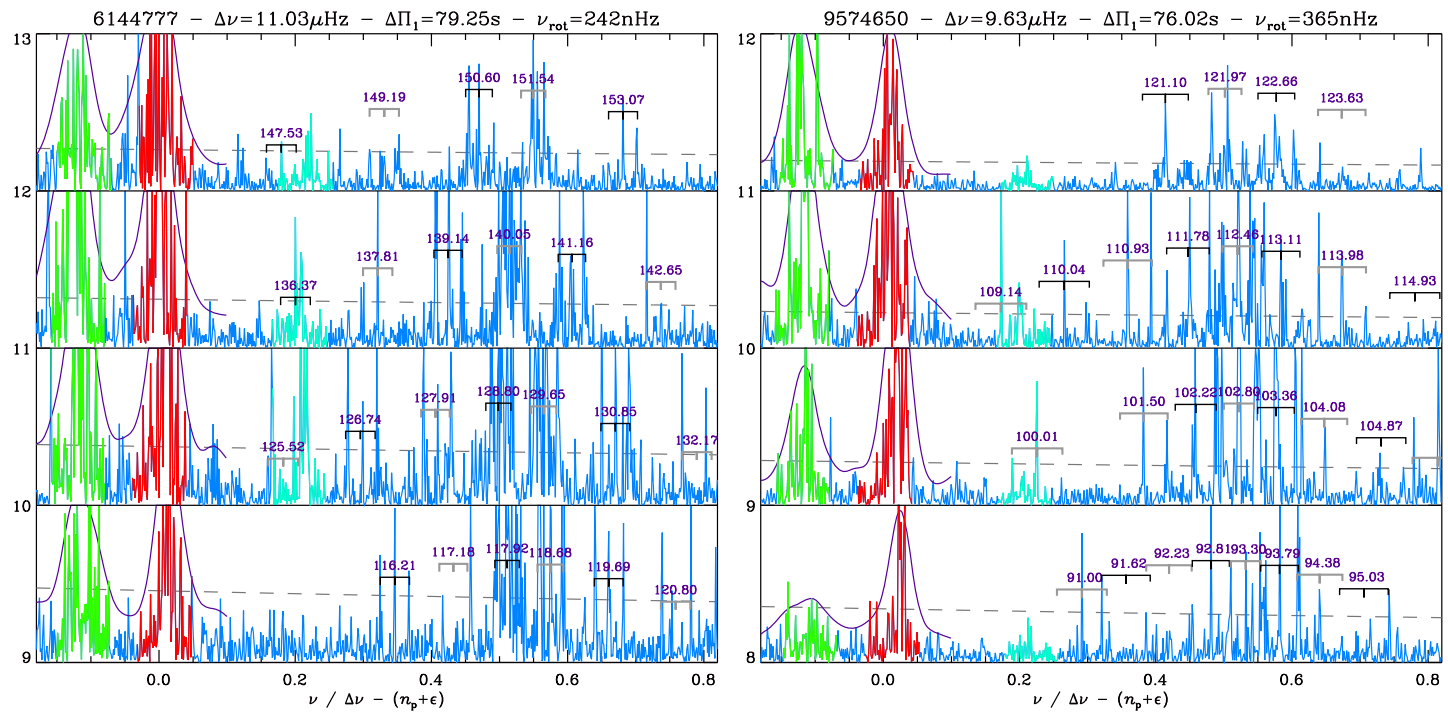

Figure 4. Zoom on the rotational splittings of the mixed modes in the giants KIC 6144777 and 9574650 , in an échelle diagram as a function of the reduced frequency $v / \Delta v-(n+\varepsilon)$. At low frequency, multiplets are overlapping. Radial and quadrupole modes, in red and green respectively, are located around the dimensionless abscissa 0 and -0.12 . The dashed lines indicate the mean value of the background multiplied by 8. From [29].
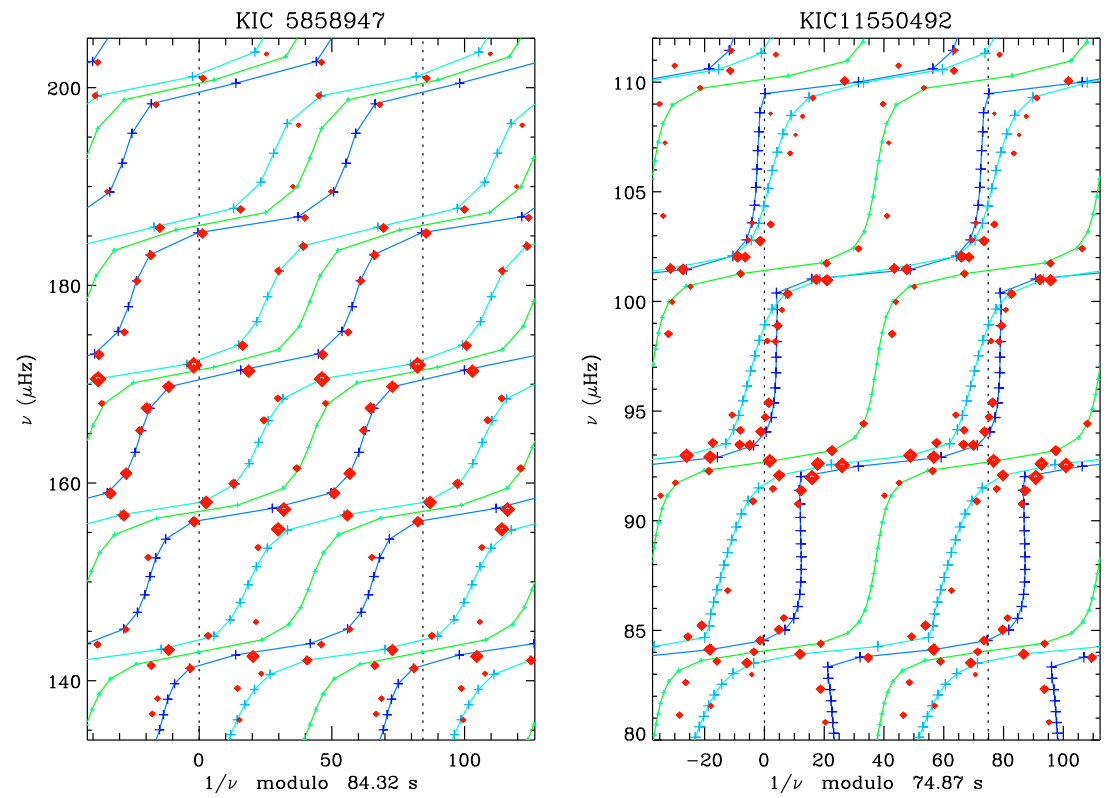

Figure 5. Gravity échelle diagrams of the two RGB stars KIC 5858947 and 11550492 . The $x$-axis is the period $1 / \nu$ modulo the gravity spacing $\Delta \Pi_{1}$; for clarity, the range has been extended from -0.5 to $1.5 \Delta \Pi_{1}$. The size of the selected observed mixed modes (red diamonds) indicates their height. Plusses give the expected location of the mixed modes, with $m=-1$ in light blue, $m=0$ in green and $m=+1$ in dark blue. 
EPJ Web of Conferences

Table 1. Asymptotic fit.

\begin{tabular}{lclcc}
\hline Modes & Method & \multicolumn{2}{c}{ Parameters } & Remark \\
& & First step & Refined step & \\
\hline Pressure modes & Universal pattern & Large separation $\Delta \nu$ & & $\varepsilon=\varepsilon(\Delta \nu) d_{01}=d_{01}(\Delta \nu)$ \\
& & & Glitch $\delta \varepsilon$ & $|\delta \varepsilon| \leq 0.02$ \\
\hline Mixed modes & Asymptotic & $\begin{array}{l}\text { Period spacing } \Delta \Pi_{1} \\
\text { Coupling factor } q\end{array}$ & $\varepsilon_{\mathrm{g}}=0$ \\
& & & $\varepsilon_{\mathrm{g}} \neq 0$ & \\
\hline Rotational splittings & Empirical & Core splittings $\delta v_{\text {rot }}$ & \\
& & & 2 parameters \\
\hline
\end{tabular}

of the core rotation occurs in red giants. This spin down, observed on the RGB but much more marked for clump stars, requires an significant angular momentum transport between the different regions of the star.

\section{FROM ASTEROSEISMIC OBSERVATIONS TO STELLAR PHYSICS}

The analysis of the thousands of red giant oscillation spectra has just started. The description of these spectra with the combination of the universal red giant oscillation pattern, the asymptotic development of mixed modes and an empirical description of the rotational splittings has proven to be fruitful. As shown in Table 1, four parameters are enough to identify all modes. Refined fits are obtained with eight free parameters, to be compared to the number of fitted modes (which amount to 40 - 140) and to the complexity of some spectra (Fig. 5).

Undoubtedly, the high-quality asteroseismic constraints, especially those probing the stellar core, are leading to great progress in stellar physics.

\subsection{Standard candles}

The precise asteroseismic constraints on red giants, and especially the precise estimate of the radius from scaling relations, completed with the more precise determination derived from stellar modeling, allows to use red giants as standard candles [40, 41]. According to Eq. (5), this requires the use of reliable effective temperatures $T_{\text {eff }}$, determined from photometry and color- $T_{\text {eff }}$ calibrations. Luminosities and distances are derived from dereddened apparent 2MASS magnitudes and bolometric corrections. Combining distances with spectroscopic constraints and asteroseismic estimates of the mass allows a detailed characterization of populations of giants in different regions of the Galaxy observed by Kepler and CoRoT at a large set of galactic latitudes and longitudes [40, 41]. This topic is more precisely developed by Miglio et al. in these proceedings.

\subsection{Modeling}

Modeling effort has been achieved for a limited number of red giants with seismic constraints [32, 4245]. If not based on grid computing, this effort is time consuming, as it addresses the physical input in the modeling. Then, it makes the best of the seismic constraints. In some stars, the lifetimes of the gravity-dominated mixed modes is so long that it exceeds the total duration of the observation run (31 months at the time this article is written), so that the accuracy of the frequency determination is equal to the frequency resolution $(\simeq 12 \mathrm{nHz})$, much better than the current performance of modeling [46]. As a consequence, future developments are very promising. 


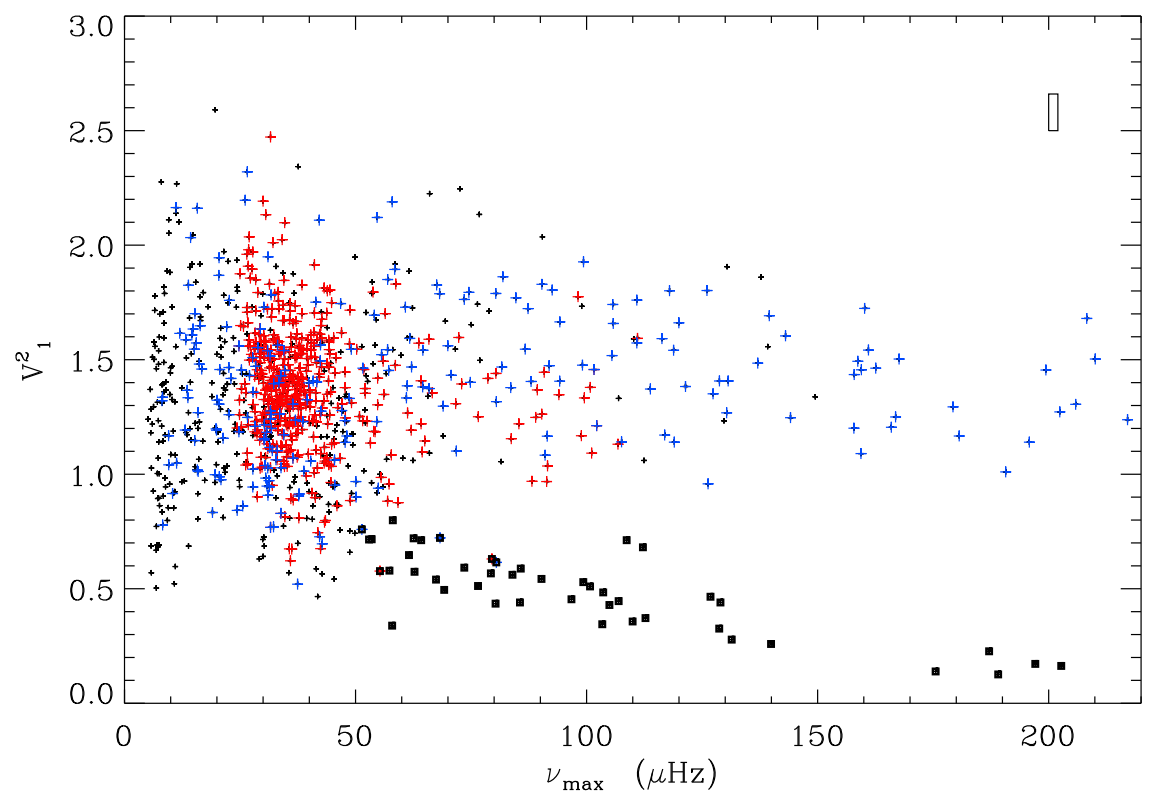

Figure 6. Visibility $V_{1}^{2}$ as a function of $v_{\max }$, with the same color code as in Fig. 2. Large black symbols indicate the population of stars with very low $V_{1}^{2}$ values.

\subsection{Low-amplitude dipole mixed modes}

Most red giants show a complex spectrum, with short-lived pressure-dominated and long-lived gravitydominated mixed modes. Bur a family of red giants shows non-standard spectra, with depressed dipole modes [23]. Such red giants are found at all evolutionary stages from the early RGB to the red clump (Fig. 6). The coupling between the two cavities, i.e. the envelope and the core, certainly obeys specific conditions that govern such a behavior. Clarifying the situation of these stars will greatly help our understanding of the mixed modes in red giants.

\subsection{Upper red giant branch; asymptotic giant branch}

Red giants ascending the RGB or the AGB have such large radii that their oscillation occur at very low frequencies, as shown by the analysis of the upper RGB from OGLE observations [47]. By extrapolation of the current results, the extension of the Kepler mission can provide us with the observation of giants with large separation as low as $0.20 \mu \mathrm{Hz}$. If the scaling relations are still valid, this corresponds to radii of about $80 R_{\odot}$, maybe not enough for investigating the tip of the RGB at all masses, but useful for combining with OGLE results.

\subsection{Differential rotation and angular momentum transport}

The observation of the rotational splittings implies that the angular momentum is, as expected, significantly redistributed between the different regions of the stars. A thorough analysis of this redistribution has just started. This will take time, but we are confident that the new constraints provided by asteroseismic observation will be translated by theoreticians into highly valuable information. 
EPJ Web of Conferences

\section{References}

[1] E. Michel, A. Baglin, M. Auvergne, C. Catala, R. Samadi, F. Baudin, T. Appourchaux, C. Barban, W.W. Weiss, G. Berthomieu et al., Science 322, 558 (2008), 0812. 1267

[2] W.J. Borucki, D. Koch, G. Basri, N. Batalha, T. Brown, D. Caldwell, J. Caldwell, J. ChristensenDalsgaard, W.D. Cochran, E. DeVore et al., Science 327, 977 (2010)

[3] J. De Ridder, C. Barban, F. Baudin, F. Carrier, A.P. Hatzes, S. Hekker, T. Kallinger, W.W. Weiss, A. Baglin, M. Auvergne et al., Nature 459, 398 (2009)

[4] S. Hekker, T. Kallinger, F. Baudin, J. De Ridder, C. Barban, F. Carrier, A.P. Hatzes, W.W. Weiss, A. Baglin, A\&A 506, 465 (2009), 0906.5002

[5] T.R. Bedding, D. Huber, D. Stello, Y.P. Elsworth, S. Hekker, T. Kallinger, S. Mathur, B. Mosser, H.L. Preston, J. Ballot et al., ApJ 713, L176 (2010), 1001.0229

[6] D. Huber, T.R. Bedding, D. Stello, B. Mosser, S. Mathur, T. Kallinger, S. Hekker, Y.P. Elsworth, D.L. Buzasi, J. De Ridder et al., ApJ 723, 1607 (2010), 1010.4566

[7] B. Mosser, K. Belkacem, M. Goupil, A. Miglio, T. Morel, C. Barban, F. Baudin, S. Hekker, R. Samadi, J. De Ridder et al., A\&A 517, A22 (2010), 1004.0449

[8] S. Frandsen, F. Carrier, C. Aerts, D. Stello, T. Maas, M. Burnet, H. Bruntt, T.C. Teixeira, J.R. de Medeiros, F. Bouchy et al., A\&A 394, L5 (2002), arXiv: astro-ph/0209325

[9] C. Barban, J.M. Matthews, J. De Ridder, F. Baudin, R. Kuschnig, A. Mazumdar, R. Samadi, D.B. Guenther, A.F.J. Moffat, S.M. Rucinski et al., A\&A 468, 1033 (2007)

[10] J. Christensen-Dalsgaard, ArXiv e-prints 1106.5946 (2011), 1106.5946

[11] J. Montalbán, A. Miglio, A. Noels, R. Scuflaire, P. Ventura, F. D’Antona, Adiabatic Solar-Like Oscillations in Red Giant Stars (2012), p. 23

[12] T.R. Bedding, ArXiv e-prints 1107.1723 (2011), 1107.1723

[13] T. Kallinger, B. Mosser, S. Hekker, D. Huber, D. Stello, S. Mathur, S. Basu, T.R. Bedding, W.J. Chaplin, J. De Ridder et al., A\&A 522, A1 (2010), 1010. 4589

[14] D. Huber, T.R. Bedding, D. Stello, S. Hekker, S. Mathur, B. Mosser, G.A. Verner, A. Bonanno, D.L. Buzasi, T.L. Campante et al., ApJ 743, 143 (2011), 1109.3460

[15] S. Hekker, Y. Elsworth, J. De Ridder, B. Mosser, R.A. García, T. Kallinger, S. Mathur, D. Huber, D.L. Buzasi, H.L. Preston et al., A\&A 525, A131 (2011), 1008. 2959

[16] B. Mosser, T. Appourchaux, A\&A 508, 877 (2009), 0909.0782

[17] I.W. Roxburgh, A\&A 506, 435 (2009)

[18] B. Mosser, Astronomische Nachrichten 331, 944 (2010), 1006. 5530

[19] A.S. Eddington, The Observatory 40, 290 (1917)

[20] T.R. White, T.R. Bedding, D. Stello, T. Appourchaux, J. Ballot, O. Benomar, A. Bonanno, A.M. Broomhall, T.L. Campante, W.J. Chaplin et al., ApJ 742, L3 (2011), 1110.1375

[21] B. Mosser, E. Michel, K. Belkacem, J. Marques, B. Mosser, O. Benomar, M. Goupil, H. Kjeldsen, T.R. Bedding, J. De Ridder et al., submitted to A\&A (2013), 1205.4023

[22] K. Belkacem, M.J. Goupil, M.A. Dupret, R. Samadi, F. Baudin, A. Noels, B. Mosser, A\&A 530, A142 (2011), 1104.0630

[23] B. Mosser, Y. Elsworth, S. Hekker, D. Huber, T. Kallinger, S. Mathur, K. Belkacem, M.J. Goupil, R. Samadi, C. Barban et al., A\&A 537, A30 (2012), 1110.0980

[24] S. Mathur, S. Hekker, R. Trampedach, J. Ballot, T. Kallinger, D. Buzasi, R.A. García, D. Huber, A. Jiménez, B. Mosser et al., ApJ 741, 119 (2011), 1109.1194

[25] D. Stello, D. Huber, T. Kallinger, S. Basu, B. Mosser, S. Hekker, S. Mathur, R.A. García, T.R. Bedding, H. Kjeldsen et al., ApJ 737, L10 (2011), 1107.0490

[26] R. Samadi, K. Belkacem, M.A. Dupret, H.G. Ludwig, F. Baudin, E. Caffau, M.J. Goupil, C. Barban, A\&A 543, A120 (2012), 1205.4846

[27] B. Mosser, K. Belkacem, M. Goupil, E. Michel, Y. Elsworth, C. Barban, T. Kallinger, S. Hekker, J. De Ridder, R. Samadi et al., A\&A 525, L9 (2011), 1011.1928 
Ageing Low Mass Stars: From Red Giants to White Dwarfs

[28] M. Tassoul, ApJS 43, 469 (1980)

[29] B. Mosser, M.J. Goupil, K. Belkacem, J.P. Marques, P.G. Beck, S. Bloemen, J. De Ridder, C. Barban, S. Deheuvels, Y. Elsworth et al., ArXiv e-prints 1209.3336 (2012), 1209. 3336

[30] E. Corsaro, D. Stello, D. Huber, T.R. Bedding, A. Bonanno, K. Brogaard, T. Kallinger, O. Benomar, T.R. White, B. Mosser et al., ApJ 757, 190 (2012), 1205.4023

[31] T. Kallinger, S. Hekker, B. Mosser, J. De Ridder, T.R. Bedding, Y.P. Elsworth, M. Gruberbauer, D.B. Guenther, D. Stello, S. Basu et al., A\&A 541, A51 (2012), 1203.3134

[32] A. Miglio, J. Montalbán, F. Carrier, J. De Ridder, B. Mosser, P. Eggenberger, R. Scuflaire, P. Ventura, F. D’Antona, A. Noels et al., A\&A 520, L6 (2010), 1009.1024

[33] J. Provost, B. Mosser, G. Berthomieu, A\&A 274, 595 (1993)

[34] P.G. Beck, T.R. Bedding, B. Mosser, D. Stello, R.A. Garcia, T. Kallinger, S. Hekker, Y. Elsworth, S. Frandsen, F. Carrier et al., Science 332, 205 (2011)

[35] T.R. Bedding, B. Mosser, D. Huber, J. Montalbán, P. Beck, J. Christensen-Dalsgaard, Y.P. Elsworth, R.A. García, A. Miglio, D. Stello et al., Nature 471, 608 (2011), 1103.5805

[36] B. Mosser, C. Barban, J. Montalbán, P.G. Beck, A. Miglio, K. Belkacem, M.J. Goupil, S. Hekker, J. De Ridder, M.A. Dupret et al., A\&A 532, A86 (2011), 1105.6113

[37] B. Mosser, M.J. Goupil, K. Belkacem, E. Michel, D. Stello, J.P. Marques, Y. Elsworth, C. Barban, P.G. Beck, T.R. Bedding et al., A\&A 540, A143 (2012), 1203.0689

[38] W. Unno, Y. Osaki, H. Ando, H. Saio, H. Shibahashi, Nonradial oscillations of stars (1989)

[39] P.G. Beck, J. Montalban, T. Kallinger, J. De Ridder, C. Aerts, R.A. García, S. Hekker, M.A. Dupret, B. Mosser, P. Eggenberger et al., Nature 481, 55 (2012), 1112 . 2825

[40] A. Miglio, J. Montalbán, F. Baudin, P. Eggenberger, A. Noels, S. Hekker, J. De Ridder, W. Weiss, A. Baglin, A\&A 503, L21 (2009), 0908.0210

[41] A. Miglio, T. Morel, M. Barbieri, B. Mosser, L. Girardi, J. Montalbán, M. Valentini, Solar-like pulsating stars as distance indicators: $G-K$ giants in the CoRoT and Kepler fields, in European Physical Journal Web of Conferences (2012), Vol. 19 of European Physical Journal Web of Conferences, p. 5012, 1111.4366

[42] F. Carrier, J. De Ridder, F. Baudin, C. Barban, A.P. Hatzes, S. Hekker, T. Kallinger, A. Miglio, J. Montalbán, T. Morel et al., A\&A 509, A73 (2010), 1001. 1655

[43] C. Jiang, B.W. Jiang, J. Christensen-Dalsgaard, T.R. Bedding, D. Stello, D. Huber, S. Frandsen, H. Kjeldsen, C. Karoff, B. Mosser et al., ApJ 742, 120 (2011), 1109.0962

[44] M.P. di Mauro, D. Cardini, G. Catanzaro, R. Ventura, C. Barban, T.R. Bedding, J. ChristensenDalsgaard, J. De Ridder, S. Hekker, D. Huber et al., MNRAS 415, 3783 (2011), 1105. 1076

[45] F. Baudin, C. Barban, M.J. Goupil, R. Samadi, Y. Lebreton, H. Bruntt, T. Morel, L. Lefèvre, E. Michel, B. Mosser et al., A\&A 538, A73 (2012), 1111.6543

[46] M. di Mauro, al., these proceedings (2013), 1205.4023

[47] W.A. Dziembowski, I. Soszyński, A\&A 524, A88 (2010), 1009.5989 